\title{
Educación de la inteligencia emocional en edad escolar: Un estudio exploratorio desde la perspectiva docente
}

\author{
Education of Emotional Intelligence at School-age: An Exploratory StudyFrom the \\ Teaching Perspective
}

\section{Educação da inteligência emocional no ensino fundamental: Um estudo exploratório a partir da perspectiva docente}

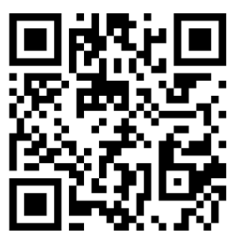

\author{
Pedro Antonio García-Tudela \\ Universidad de Murcia \\ Murcia, España \\ pedroantonio.garcia4@um.es \\ https://orcid.org/0000-0003-0405-923X \\ Pedro Marín-Sánchez \\ Consejería de Educación de la Región de Murcia \\ Murcia, España \\ pedro.marin4@murciaeduca.es \\ https://orcid.org/0000-0003-4444-081X
}

Recibido • Received • Recebido: 04 / 10 / 2019

Corregido • Revised • Revisado: 21 / 05 / 2021

Aceptado • Accepted $\cdot$ Aprovado: 07 / $06 / 2021$

\begin{abstract}
Resumen
Introducción. En la actualidad existe una tendencia educativa hacia una pedagogía afectiva. Por ello, en este trabajo se investiga el conocimiento y la experiencia que docentes de educación primaria (alumnado de entre 6 y 12 años) tienen sobre la inteligencia emocional y su educación. Metodología. Un total de 100 docentes de España participan en este estudio transaccional exploratorio. Concretamente, la muestra conseguida a través de una técnica no probabilística de tipo casual ha respondido un cuestionario a partir del que se ha realizado un análisis de datos cuantitativos y cualitativos a través de SPSS y Atlas.ti. Resultados. En relación con los conocimientos teóricos sobre el objeto de estudio, se han elaborado 35 códigos donde se recogen 236 citas textuales; no se haya ninguna correlación positiva entre el trabajo cotidiano de la IE en el aula y las diferentes variables sociodemográficas; los datos también evidencian que existen centros educativos en los que se implementan programas de inteligencia emocional para todos los cursos, en cambio, en otros centros se halla cierta incoherencia interna. Por último, existe una significativa predisposición a formarse en la educación emocional. Discusión y conclusiones. Una destacable parte de la muestra tiene una adecuada visión teórica del concepto de la inteligencia emocional según los autores de referencia. Sin embargo, a pesar de existir una elevada implicación práctica, en muchas ocasiones se trabaja de una manera informal o no explícita, cuando el reto actual consiste en desarrollar programas longitudinales. Se proponen nuevas líneas de investigación vinculadas a los hallazgos de este estudio, como el desarrollo de un estudio descriptivo y otro experimental.
\end{abstract}

Palabras claves: Inteligencia emocional; educación primaria; desarrollo de habilidades; docentes; investigación exploratoria. 
http://doi.org/10.15359/ree.25-3.6

http://www.una.ac.cr/educare

educare@una.ac.cr

\begin{abstract}
:
Introduction. Currently, there is an educational trend towards an affective pedagogy. For this reason, this paper investigates the knowledge and experience that Primary Education teachers (students between 6 and 12 years old) have on emotional intelligence and their education. Methodology. One hundred teachers from Spain participate in this exploratory transactional study. Specifically, the sample obtained through a random non-probabilistic technique has answered a questionnaire from which an analysis of quantitative and qualitative data has been carried out through SPSS and Atlas. ti. Results. Concerning the theoretical knowledge about the object of study, 35 codes have been elaborated where 236 textual citations are collected; there is no positive correlation between the daily work of El in the classroom and the different sociodemographic variables. Data also show that there are educational centers in which emotional intelligence programs are implemented for all courses; on the other hand, there is some internal incoherence in other centers. Finally, there is a significant predisposition to be trained in emotional education. Discussion and Conclusions. According to the reference authors, a remarkable part of the sample has an adequate theoretical vision of the concept of emotional intelligence. However, despite the existence of high practical involvement, it is often worked in an informal or not explicit way when the current challenge consists of developing explicit programs. New lines of research linked to the findings of this study are proposed, such as the development of a descriptive and an experimental study.
\end{abstract}

Keywords: Emotional intelligence; primary education; skill development; teachers; exploratory investigation.

\title{
Resumo:
}

Introdução. Atualmente existe uma tendência educacional para uma pedagogia afetiva. Por esse motivo, este trabalho investiga o conhecimento e a experiência que o corpo docente de Ensino Fundamental (estudantes entre 6 e 12 anos) têm sobre inteligência emocional e sua formação. Metodologia. Um total de 100 professores da Espanha participaram deste estudo transacional exploratório. Especificamente, a amostra obtida por meio de técnica aleatória não probabilística do tipo causal respondeu a um questionário a partir do qual foi realizada uma análise de dados quantitativos e qualitativos por meio do SPSS e Atlas.ti. Resultados. Em relação ao conhecimento teórico sobre o objeto de estudo, foram elaborados 35 códigos onde são coletadas 236 citações textuais; não há correlação positiva entre o trabalho diário da IE em sala de aula e as diferentes variáveis sociodemográficas; Os dados também mostram que existem centros educacionais nos quais são implantados programas de inteligência emocional para todos os cursos, por outro lado, nos demais centros há alguma incoerência interna. Finalmente, existe uma predisposição significativa para ser treinado em educação emocional. Discussão e conclusões. Uma parte notável da amostra possui uma visão teórica adequada do conceito de inteligência emocional segundo os autores de referência. No entanto, apesar da existência de elevado envolvimento prático, em muitas ocasiões é trabalhado de forma informal ou não explícita, quando o desafio atual consiste em desenvolver programas explícitos. São propostas novas linhas de pesquisa vinculadas aos achados deste estudo, como o desenvolvimento de um estudo descritivo e outro experimental.

Palavras-chave: Inteligência emocional; ensino fundamental; desenvolvimento de habilidades; corpo docente; investigação exploratória. 
http://doi.org/10.15359/ree.25-3.6

\section{Introducción}

Actualmente, a pesar de las diferentes transformaciones sociales y las reformas legales que se han llevado a cabo en el campo de la educación, se continúa velando por un proceso de enseñanza-aprendizaje cuyo principal interés es el bienestar personal a partir del desarrollo de diferentes habilidades como la socialización, la empatía, etc. Por lo que se podría afirmar que prima una pedagogía afectiva (Uitto et al., 2015).

La respuesta educativa para desarrollar las diferentes habilidades y así conseguir afrontar la vida con una mayor probabilidad de éxito se denomina educación emocional. De esta forma, se educan las competencias básicas para la vida (regulación emocional, autonomía emocional, entre otras) que favorecen el bienestar personal y colectivo (Bisquerra Alzina, 2011).

Debido al interés social y a la implicación pedagógica que en estos últimos años está adquiriendo la Inteligencia Emocional (IE a partir de ahora), la presente investigación persigue conocer y analizar los conocimientos que tiene el personal docente de Educación Primaria (en el contexto español de los 6 a los 12 años) en torno a este objeto de estudio, así como las propuestas escolares que diseñan e implementan en sus contextos educativos.

\section{Marco teórico}

\section{En torno al concepto y los modelos de inteligencia emocional}

El objetivo de este trabajo no consiste en establecer una discusión técnica y más propia de la neurociencia sobre las emociones. No obstante, es preciso partir del hecho de que una emoción se define como una reacción conductual y subjetiva a causa de un estímulo externo o interno (memoria) que destaca por su espontaneidad y su reflejo facial y corporal (Mora, 2013).

Además, es preciso señalar la existencia de la discusión terminológica en torno al binomio emoción-sentimiento. Principalmente, se ha señalado que el sentimiento es el etiquetado consciente de una emoción una vez que el sujeto reconoce las sensaciones que experimenta ante un estímulo, originándose de esta forma un proceso cognitivo como repercusión de la experiencia vivenciada (Damasio, 1994). Asimismo, también se ha destacado que existe una ambigüedad referencial en cuanto al sentimiento como componente sensorial o como estado mental compuesto por elementos afectivos, cognitivos, etc. (Rosas, 2011).

Tal y como se ha indicado, la discusión conceptual expuesta se aleja del campo de interés de las ciencias de la educación y, por lo tanto, del interés de este trabajo. En cambio, abordar el concepto de la IE recobra más sentido en el contexto escolar. En relación con la primera definición que se hizo, esta significó el punto de inflexión a partir del cual comenzó a desarrollarse la pedagogía de la emoción. Concretamente, Salovey y Mayer (1990) definieron dicho constructo como el conjunto de habilidades y subhabilidades que conllevan procesos psicosociales vinculados a la individualidad de la persona 
http://doi.org/10.15359/ree.25-3.6

http://www.una.ac.cr/educare

educare@una.ac.cr

Posteriormente, se complementó la definición original y se profundizó en el hecho de que la IE implica una percepción, asimilación, comprensión y regulación de las emociones, las cuales son practicables para así potenciarlas (Mayer y Salovey, 1997).

Otra de las definiciones clásicas de este concepto es la realizada por Goleman (1995): capacidad de reconocer nuestros propios sentimientos y los de los demás, de motivarnos y de manejar adecuadamente las relaciones. Sin embargo, tres años más tarde también redefinió su primera conjetura, aunque sin aplicar grandes cambios respecto a la original, simplemente enfatizando la idea de gestionar las emociones en la propia persona misma y en las relaciones interpersonales (Goleman, 1998).

Además, también existen otras definiciones sobre la IE que se construyen a partir del análisis de otras clásicas como las expuestas en este trabajo. Concretamente, García-Fernández y Giménez-Mas (2010) afirman que "es la capacidad que tiene el individuo de adaptarse e interaccionar con el entorno dinámico y cambiante a raíz de sus propias emociones" (p. 45). Asimismo, la completan añadiendo que engloba diferentes habilidades como la autoconciencia, perseverancia, la motivación, entre otras.

Las definiciones sobre este concepto son una aproximación a los modelos teóricos de $\mathrm{IE}$, los cuales aún poseen un mayor interés científico, puesto que exponen un constructo más complejo a partir del cual definir sus principales componentes.

Uno de los modelos más populares y utilizados son por ejemplo el de Mayer y Salovey (1997), el cual se basa en cuatro ramas o áreas de capacidades o habilidades de la IE: percibir las emociones propias y del resto, emplear las emociones para facilitar el pensamiento, comprender los significados emocionales y controlar las emociones.

Concretamente, el modelo previamente mencionado, se define como un modelo basado en habilidades. Sin embargo, otros como el de Goleman (1995) es de tipo mixto, puesto que también incluye rasgos de la personalidad. De una manera más específica, este modelo se basa en los siguientes componentes: conciencia de la propia persona autorregulación, motivación, empatía y habilidades sociales (Goleman, 1995). El modelo de Bar-On (2006) también se considera un modelo mixto, el cual se articula en torno a diferentes componentes, algunos de ellos son el intrapersonal (comprensión emocional, autorrealización, independencia, etc.), la adaptabilidad (solución de problemas, flexibilidad, etc.), el de manejo del estrés (tolerancia del estrés y control de los impulsos), entre otros.

Teniendo en cuenta la posibilidad de implementar un modelo de IE en un contexto concreto (una empresa, una asociación, una clase de secundaria, etc.) existe una nueva categoría de modelos que contiene aquellos que incluyen componentes de personalidad, habilidades y otros factores. Expresado de otro modo, modelos divididos en grandes dimensiones que 
http://doi.org/10.15359/ree.25-3.6

recogen diferentes tipos de habilidades, tanto intrapersonales, como interpersonales y que se ajustan a las necesidades o demandas del público objetivo. García-Fernández y Giménez-Mas (2010) exponen diferentes ejemplos referentes a esta clasificación, como el modelo de Cooper y Sawaf para el ámbito organizacional de la empresa (García-Fernández y Giménez-Mas, 2010). No obstante, dentro de esta clasificación de otros modelos también mencionan ejemplos que tratan de enfatizar la divulgación de la IE, como el modelo de Rovira (1998) dividido en 12 dimensiones (actitud positiva, ser capaz de superar dificultades, reconocer las propias emociones, etc.).

En relación con la categoría de modelos vinculados a un público objetivo, recientemente también se han generado modelos de IE con una orientación exclusivamente educativa para el contexto formal. De una manera más específica, un ejemplo de ello es el modelo zeta propuesto por García-Tudela y Marín-Sánchez (2019). Principalmente se sustenta en tres bloques de habilidades. El primero relacionado con el propio estudiantado (bloque intra-emocional: autorregulación, autoconfianza, autoconocimiento emocional, etc.), el segundo bloque consiste en las habilidades sociales que se ponen en práctica al estar e interactuar con otras personas (bloque inter-emocional: asertividad, empatía, cooperatividad, etc.) y por último, un bloque que acentúa la vinculación del estudiantado con la sociedad, su contexto y la cultura (bloque socio-emocional: colaboración intercultural, compromiso proambiental, etc.).

\section{La educación de la inteligencia emocional}

A pesar de las posibilidades que la IE puede brindar al alumnado, a las familias y al personal docente (Casino-García et al., 2021; Chen y Guo, 2020), existen trabajos como el de García-Retana (2012) a través del que se reflexiona sobre cómo, a lo largo del tiempo, ha existido en el contexto escolar una tendencia por priorizar el componente cognitivo al emocional. Sin embargo, son numerosos los centros educativos de enseñanzas obligatorias que trabajan la IE dentro de sus jornadas escolares.

De una manera generalista, se podría afirmar que los objetivos al aplicar un programa de IE son similares independientemente del nivel educativo. Principalmente, se persigue mejorar las habilidades socioemocionales del alumnado para así, optimizar la convivencia escolar y el bienestar personal (Galdón López y Melguizo Ibañez, 2019; Pastor-Gil y Blázquez-Saiz, 2019; Salas et al., 2018).

Como se ha indicado, los objetivos son comunes, pero diferente la manera de alcanzarlos. Generalmente, cabe destacar que en educación primaria existe una tendencia a desarrollar actividades relacionadas con la IE a través de técnicas propias del aprendizaje cooperativo, de las cuales se han obtenido resultados positivos durante los últimos años (Rivera-Pérez et al., 2021; Torrego-Seijo et al., 2020). Asimismo, son diferentes las propuestas de IE que se han desarrollado en primaria a partir de metodologías o estrategias lúdicas como la gamificación 
http://doi.org/10.15359/ree.25-3.6

http://www.una.ac.cr/educare

educare@una.ac.cr

(Pozo-Rico y Sandoval, 2020) o el aprendizaje basado en juegos (Dell'Angela et al., 2020; Hassan et al., 2021). También destacan otros proyectos basados principalmente en el teatro, debates, proyección de vídeos, etc. (Gómez Vicario et al., 2017; Morán Iranzo et al., 2016).

De manera complementaria a estos hallazgos, también cabe destacar que en algunos casos la IE no se trabaja de una manera puntual en el aula de primaria, sino que comprende programas educativos extendidos en el tiempo (Vázquez y López-Larrosa, 2016); también se aprecia como una oportunidad clave para algunas asignaturas concretas como la Educación Física (Sánchez Ortega y Chacón-Cuberos, 2021); o en otros casos, se trabaja de manera extraescolar por medio de talleres virtuales (Fernández-Díez et al., 2017).

Parte de los textos científicos, referidos a la IE y su educación, evidencian que conseguir unos resultados positivos en el aula no depende únicamente del programa aplicado, de la metodología utilizada o de los recursos empleados, sino que un elemento clave es la formación del profesorado. El personal docente que posee una elevada IE no solo enseña de una manera más eficaz y comprende mejor las necesidades académicas de su alumnado, sino que también es capaz de influir de mejor manera en crear un óptimo ambiente de clase, gestionar adecuadamente los problemas que sucedan, entre otras posibilidades para mejorar el bienestar escolar (Alam y Ahmad, 2018; Valente y Lourenço, 2020).

En cuanto al problema de investigación, este tiene dos vertientes. Por un lado, conocer si el personal docente de educación primaria comparte a nivel teórico la misma concepción sobre la IE. Y seguidamente, saber de qué manera favorece el desarrollo de las habilidades socioemocionales de sus estudiantes. En forma de pregunta de investigación sería: ¿qué conocimientos y experiencias didácticas tiene el profesorado de educación primaria sobre la inteligencia emocional?

Para resolver dicha cuestión, a través de esta investigación se persiguen los siguientes objetivos:

- Estudiar los conocimientos teóricos y las experiencias didácticas del profesorado de educación primaria sobre la inteligencia emocional.

- Detectar las similitudes y diferencias en torno al concepto de inteligencia emocional que tiene el profesorado de educación primaria.

- Conocer las principales iniciativas implementadas en el nivel de educación primaria sobre la inteligencia emocional.

- Descubrir si existe una implicación a nivel de centro educativo a través de programas o proyectos sobre inteligencia emocional. 
http://doi.org/10.15359/ree.25-3.6

\section{Método}

\section{Participantes}

La población de la presente investigación está basada en docentes de educación primaria de una ciudad de la Región de Murcia, (España) $(\mathrm{N}=113)$. Sin embargo, la muestra participante está compuesta por 100 profesionales, 68 pertenecen a centros educativos públicos y 32 a centros educativos concertados.

En cuanto a la representatividad de la muestra, se ha contemplado un nivel de confianza del $95 \%$ y un margen de error del 5\%. Por lo tanto, el tamaño muestral debería ser como mínimo de 88 sujetos. Es por ello que, teniendo en cuenta los datos de la muestra estimada y la muestra participante, se debe afirmar que la muestra de este estudio es representativa.

En relación con la técnica de muestreo realizada, se ha utilizado un muestreo no probabilístico de tipo casual o también denominado, muestreo por accesibilidad o por conveniencia. Principalmente, se ha escogido este tipo de muestreo por las razones justificadas por Sabariego Puig (2004): una muestra compuesta por individuos a los que se tiene facilidad de acceso, que se ofrecen a participar por voluntad propia y, además, señala que es uno de los muestreos más frecuentes en investigación educativa.

A continuación, en la Tabla 1 se expone la distribución de la muestra atendiendo a diferentes variables sociodemográficas como el sexo, la edad, la especialidad educativa, entre otras.

Tabla 1: Distribución de la muestra

\begin{tabular}{ccc}
\hline Variables & Respuestas & $\mathrm{n}$ \\
\hline \multirow{2}{*}{ Sexo } & Mujer & 58 \\
& Hombre & 42 \\
\hline \multirow{3}{*}{ Edad } & $24-34$ & 35 \\
& $34-44$ & 38 \\
& $44-54$ & 13 \\
& $54-64$ & 5 \\
& NS/NC & 9 \\
M & 38,27 \\
& DT & 8,88 \\
\hline
\end{tabular}


http://doi.org/10.15359/ree.25-3.6

http://www.una.ac.cr/educare

educare@una.ac.cr

\begin{tabular}{|c|c|c|}
\hline Variables & Respuestas & $\mathrm{n}$ \\
\hline \multirow{9}{*}{ Centro educativo } & Colegio 1 & 2 \\
\hline & Colegio 2 & 21 \\
\hline & Colegio 3 & 12 \\
\hline & Colegio 4 & 8 \\
\hline & Colegio 5 & 11 \\
\hline & Colegio 6 & 4 \\
\hline & Colegio 7 & 9 \\
\hline & Colegio 8 (concertado) & 15 \\
\hline & Colegio 9 (concertado) & 21 \\
\hline \multirow{9}{*}{ Especialidad } & Tutor/a & 47 \\
\hline & Inglés & 14 \\
\hline & Francés & 3 \\
\hline & Música & 5 \\
\hline & E. Física & 12 \\
\hline & Audición y Lenguaje & 5 \\
\hline & Pedagogía Terapéutica & 4 \\
\hline & Religión & 1 \\
\hline & NS/NC & 9 \\
\hline \multirow{10}{*}{ Cursos impartidos } & $1 .^{\circ}$ & 6 \\
\hline & $2 .^{\circ}$ & 7 \\
\hline & $3 .^{\circ}$ & 6 \\
\hline & $4 .^{\circ}$ & 7 \\
\hline & $5 .^{\circ}$ & 5 \\
\hline & $6 .^{\circ}$ & 9 \\
\hline & $1 .^{\circ}, 2 .^{\circ}$ y $3 .^{\circ}$ & 15 \\
\hline & $4 .^{\circ}, 5{ }^{\circ}, y 6 .^{\circ}$ & 19 \\
\hline & En todos los cursos & 18 \\
\hline & NS/NC & 8 \\
\hline
\end{tabular}

Nota: Elaboración propia. 
http://doi.org/10.15359/ree.25-3.6

\section{Metodología}

Tal y como se ha evidenciado en la revisión teórica inicial, son diversos los estudios que analizan la IE docente o la implementación concreta de una iniciativa educativa. En cambio, hay una ausencia de trabajos científicos que investiguen los conocimientos teóricos sobre la IE desde la perspectiva profesional educativa, que identifiquen y correlacionen diferentes propuestas didácticas implementadas en el nivel deeducación primaria, asícomo que analicen diferentes programas, planes y proyectos de IE. Por lo tanto, partiendo del vacío científico en torno a los objetos de estudio de este trabajo, tiene sentido definir esta investigación como un estudio transaccional exploratorio. Según Hernández Sampieri et al. (2010), este tipo de estudios son la base de posteriores investigaciones correlacionales, explicativas, etc., ya que se utilizan para examinar un tema poco estudiado. Por lo tanto, estos adquieren un gran valor, puesto que favorecen la aproximación a nuevos problemas, establecen nuevas prioridades y sugerencias para futuras líneas de investigación.

\section{Instrumento}

En referencia a la recolección de datos, esta ocurre en un único momento a través de un cuestionario ad hoc compuesto por preguntas cerradas y abiertas.

Para el diseño del cuestionario se han seguido las recomendaciones expuestas por Torrado Fonseca (2009) y Meneses (2016) referentes al aspecto, la redacción, la distribución del contenido, etc. De tal forma, que el instrumento comienza con tres preguntas sociodemográficas referentes a la edad, especialidad educativa y nivel en el que el sujeto entrevistado imparte clase. Seguidamente, tres preguntas dicotómicas para introducir el tema y tres preguntas abiertas para profundizar y obtener claves de interés para responder el problema de investigación. En la Tabla 2 se exponen las diferentes preguntas según su naturaleza.

Tabla 2: Preguntas de la entrevista

\begin{tabular}{|c|c|c|}
\hline N. ${ }^{\circ}$ & Tipo de pregunta & Pregunta \\
\hline 1 & Abierta & ¿Qué entiende usted por inteligencia emocional? \\
\hline 2 & Dicotómica y abierta & $\begin{array}{l}\text { ¿Integra la educación en inteligencia emocional en su trabajo diario en el aula? } \\
\text { En caso afirmativo, explique de qué forma; y en caso negativo, exponga las } \\
\text { principales razones. }\end{array}$ \\
\hline 3 & Dicotómica y abierta & $\begin{array}{l}\text { ¿Se establecen desde el centro escolar planes, proyectos o programas para el } \\
\text { desarrollo de la inteligencia emocional en el estudiantado? } \\
\text { En caso afirmativo, explique cómo. }\end{array}$ \\
\hline 4 & Dicotómica & $\begin{array}{l}\text { ¿Estaría interesado en formarse sobre la inteligencia emocional y su educación } \\
\text { en primaria? }\end{array}$ \\
\hline
\end{tabular}

Nota: Elaboración propia. 
http://doi.org/10.15359/ree.25-3.6

http://www.una.ac.cr/educare

educare@una.ac.cr

En cuanto a la validación del instrumento diseñado, se debe destacar que se ha seguido un procedimiento cualitativo a través de entrevistas cognitivas. Concretamente, estas permiten deducir el modo en el que se comportan las preguntas $y$, por lo tanto, analizar cuáles son sus principales problemas a nivel interpretativo (Caicedo Cavagnis y Zalazar-Jaime, 2018). Para todas las entrevistas cognitivas se ha utilizado la técnica de pensar en voz alta, es decir, la persona entrevistada debe verbalizar todos sus pensamientos mientras que está respondiendo los diferentes ítems (Collins, 2003).

La muestra utilizada para el proceso descrito estuvo compuesta por 8 docentes del nivel educativo investigado. De tal forma que, dicha representación quedó excluida de la muestra a la que se le aplicó el cuestionario final. Teniendo en cuenta que es una técnica cualitativa, la representatividad no es un factor clave, por lo que se ha tenido en cuenta la saturación teórica, es decir, se seleccionaron participantes para la validación hasta que no se obtuvieron nuevos descubrimientos (Caicedo Cavagnis y Zalazar-Jaime, 2018).

El plan de análisis de los resultados responde a una naturaleza mixta. Principalmente, se ha utilizado el programa informático SPSS (V.22) para analizar los datos derivados de las preguntas dicotómicas; mientras que, por otro lado, se ha utilizado el programa Atlas.ti (V.8) para codificar, clasificar y exponer los datos cualitativos.

Sobre el análisis cualitativo, se debe destacar que se ha seguido el proceso desarrollado por Massot et al. (2016), es decir, una vez que se han recogido los datos, estos se han registrado, se han reducido en forma de códigos y se han representado gráficamente.

\section{Resultados}

En primer lugar, los resultados relacionados con la definición que los sujetos docentes han expresado sobre la IE se recogen en forma de red semántica (Figura 1). A partir de los datos recogidos, se ha considerado pertinente crear dos códigos libres titulados definida como y útil para. En relación con el primer código libre, se han creado 12 códigos (categorías) donde quedan representadas 134 citas textuales. Por otro lado, el segundo código libre tiene asociados 23 códigos que incluyen 102 citas.

Principalmente, la IE queda definida como una capacidad, ya que es el código más utilizado $(n=50)$. Seguidamente, la IE también se define como el desarrollo de dos habilidades concretas, la gestión emocional $(n=28)$ y la empatía $(n=27)$. En cambio, es menor el número de veces que se define como habilidad/es (19). Por último, de manera minoritaria, queda definida como el motor del resto de inteligencias, una capacidad que tiene quien educa y otras definiciones con una visión más reduccionista como el trabajo de las emociones. 


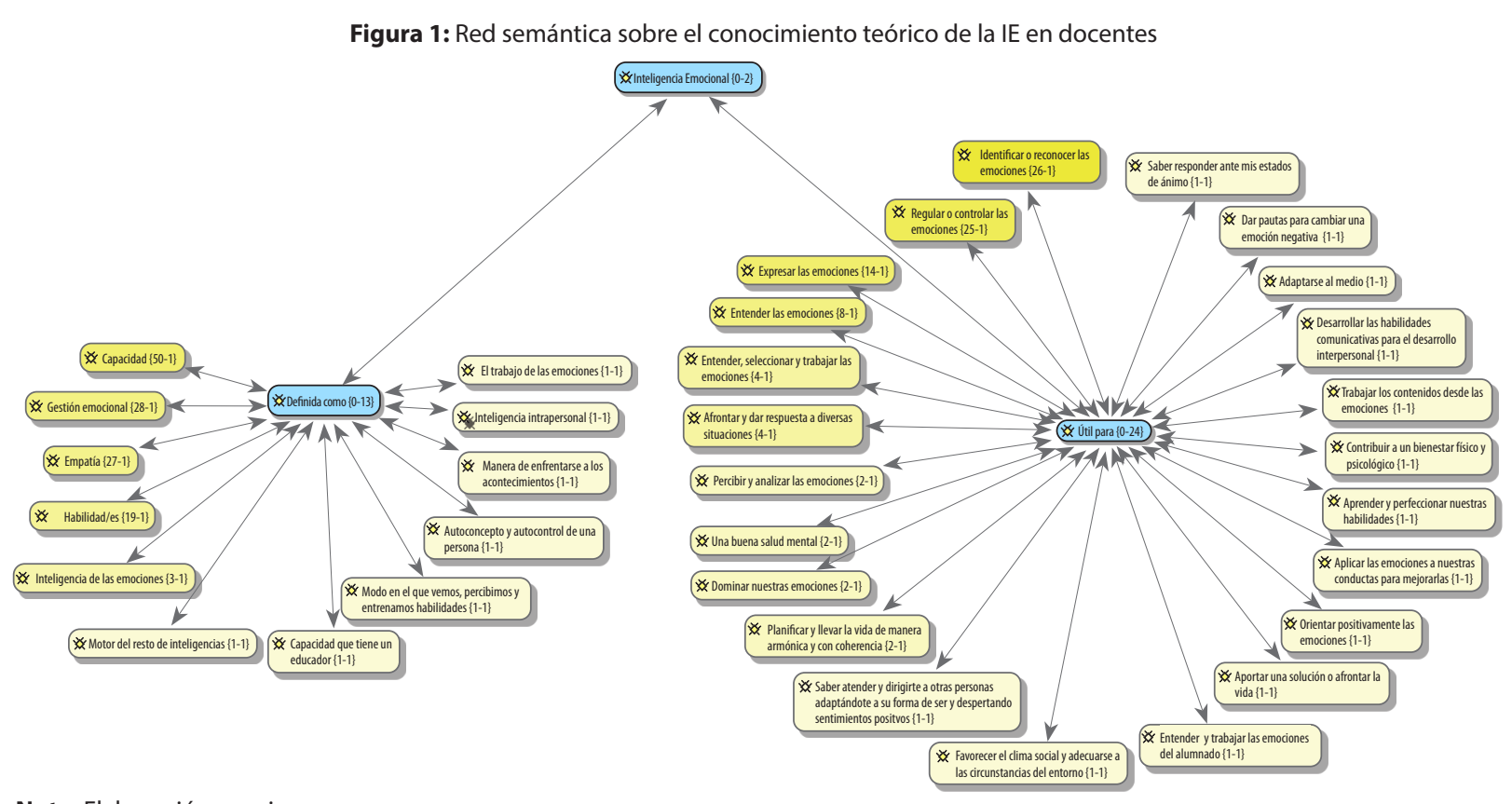

Nota: Elaboración propia.

En cuanto a la utilidad de la IE, la gran mayoría de respuestas coinciden en identificar o reconocer las emociones $(n=26)$ y regular o controlar las emociones $(n=25)$. De manera más minoritaria, expresar las emociones $(\mathrm{n}=14)$. Por último, de manera aislada destaca una buena salud mental $(n=2)$; planificar y llevar la vida de manera armónica y con coherencia $(n=2)$, contribuir a un bienestar físico y psicológico $(n=1)$, entre otras.

Profundizando en la educación de la IE, es decir, en el trabajo cotidiano que se hace con estudiantes de primaria sobre sus habilidades y emociones, se debe destacar que una amplia mayoría de la muestra ( $[n=92)$ afirma que sí trabaja la IE habitualmente con su alumnado.

Al cruzar los datos de esta pregunta dicotómica con algunas de las variables sociodemográficas iniciales, se obtienen resultados que muestran la no existencia de diferencias significativas en cuanto al trabajo cotidiano de la IE y el sexo de la persona que imparte clase (Tabla 3). Lo mismo sucede con el resto de las variables, es decir, edad, especialidad y nivel educativo.

Tabla 3: Trabajo de la IE según docente hombre o mujer

\begin{tabular}{ccccc}
\hline & & \multicolumn{3}{c}{ Trabajo diario en el aula } \\
\cline { 2 - 5 } & & Sí & No & Total \\
\hline Sexo & M & 53 & 5 & 58 \\
& H & 39 & 3 & 42 \\
\hline Total & & 92 & 8 & 100 \\
\hline
\end{tabular}

Nota: Elaboración propia. 
http://doi.org/10.15359/ree.25-3.6

http://www.una.ac.cr/educare

educare@una.ac.cr

Seguidamente y en relación absoluta con la educación de la IE, se ha generado otra red semántica en torno a la pregunta abierta sobre cómo esta se trabaja habitualmente en el aula (Figura 2).

En este caso también se origina a partir de dos códigos libres titulados metodologías o estrategias y materiales.

En referencia al primero, es decir, qué metodologías se emplean para educar la IE, cabe destacar que las 4 respuestas más repetidas han sido: en asamblea $(n=20)$, estrategias de resolución de conflictos $(n=15)$, empatizar $(n=13)$ y trabajando habilidades $(n=12)$.

En menor medida se especifica que es a través de un programa o proyecto: proyectos de interioridad $(n=4)$, a través de programas para escolares con TEA $(n=1)$. No destaca un alto grado de especificidad en cuanto a las estrategias, metodologías, o incluso actividades desarrolladas. Los únicos códigos más concretos que se han generado son: trabajando canciones $(n=4)$, teatro $(n=4)$, utilizando el humor $(n=1)$ y rutinas de pensamiento $(n=1)$.

Atendiendo al segundo código libre, los materiales que más se utilizan para educar la IE en primaria son: cuentos $(n=13)$, juegos $(n=5)$, caja de emociones $(n=4)$, vídeos $(n=4)$, aunque en menor medida también hay otras respuestas como emocionómetro $(n=2)$, fichas $(n=1)$ e internet $(\mathrm{n}=1)$.

Figura 2: Red semántica de estrategias y materiales para la didáctica de la IE

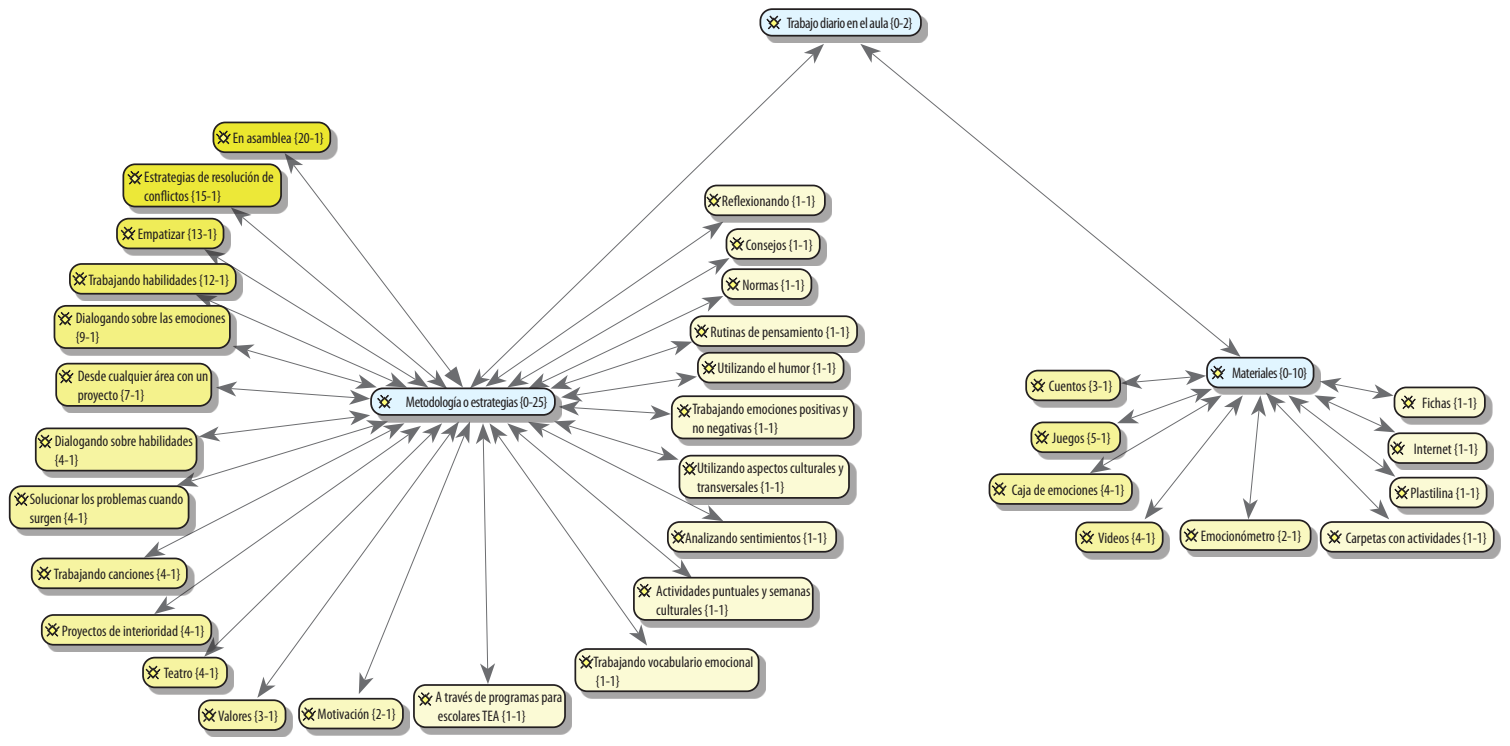

Nota: Elaboración propia. 
http://doi.org/10.15359/ree.25-3.6

En cuanto a los programas, proyectos o planes que existen a nivel de centro educativo para educar la IE en todos los cursos, cabe destacar que se han generado 30 códigos (Figura 3). Los más frecuentes son proyecto en ti $(n=14)$ y el plan de salud en la escuela $(n=13)$. Seguidamente, 9 códigos, cuya representación es de entre 3 y 5 docentes. Y, por último, 19 códigos que recogen la información emitida por 2 o menos docentes.

Figura 3: Red semántica de iniciativas nivel de centro educativo

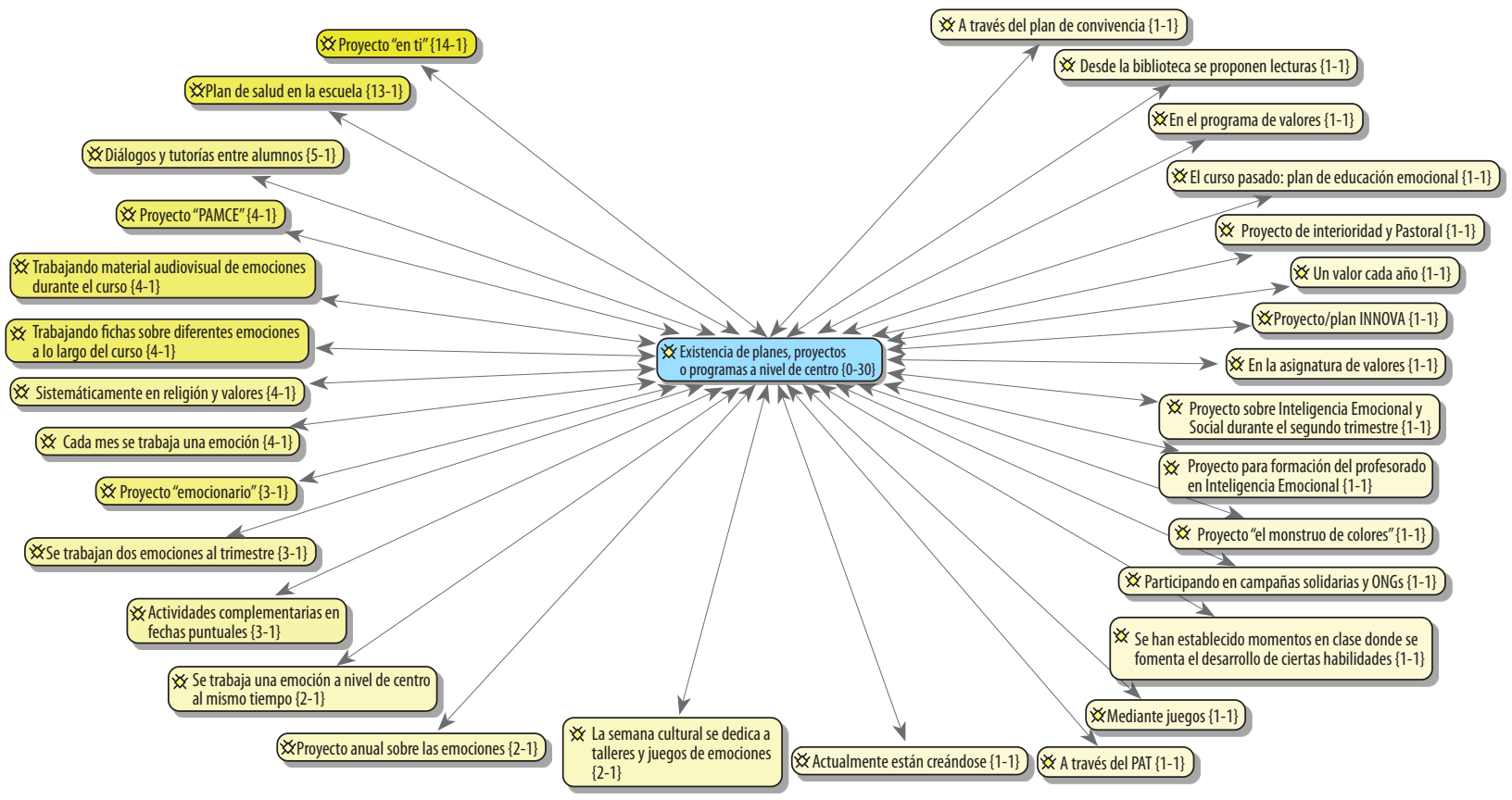

Nota: Elaboración propia.

Al analizar en términos cuantitativos la implicación del centro escolar en su conjunto, se halla que existe una baja coherencia interna, puesto que se observan diferencias destacables en los colegios 3, 4 y 8 . Concretamente, en los casos señalados existe una parte de la muestra que afirma que sí existen iniciativas a nivel de centro, mientras que el resto opina lo contrario (Tabla 4).

Debido a que la prueba de significación de chi-cuadrado indica que el valor de la significación es menor que 0.05, cabe afirmar que las diferencias extraídas son significativas.

Por último, la extensa mayoría de la muestra $(n=95)$ afirma que está interesada en recibir formación sobre cómo educar la IE de una manera cotidiana con su alumnado. Por otro lado, las cinco personas que han seleccionado la opción negativa justifican que ya están formándose en esta temática. 
http://doi.org/10.15359/ree.25-3.6

http://www.una.ac.cr/educare

educare@una.ac.cr

Tabla 4: Iniciativas a nivel de centro en relación con cada colegio

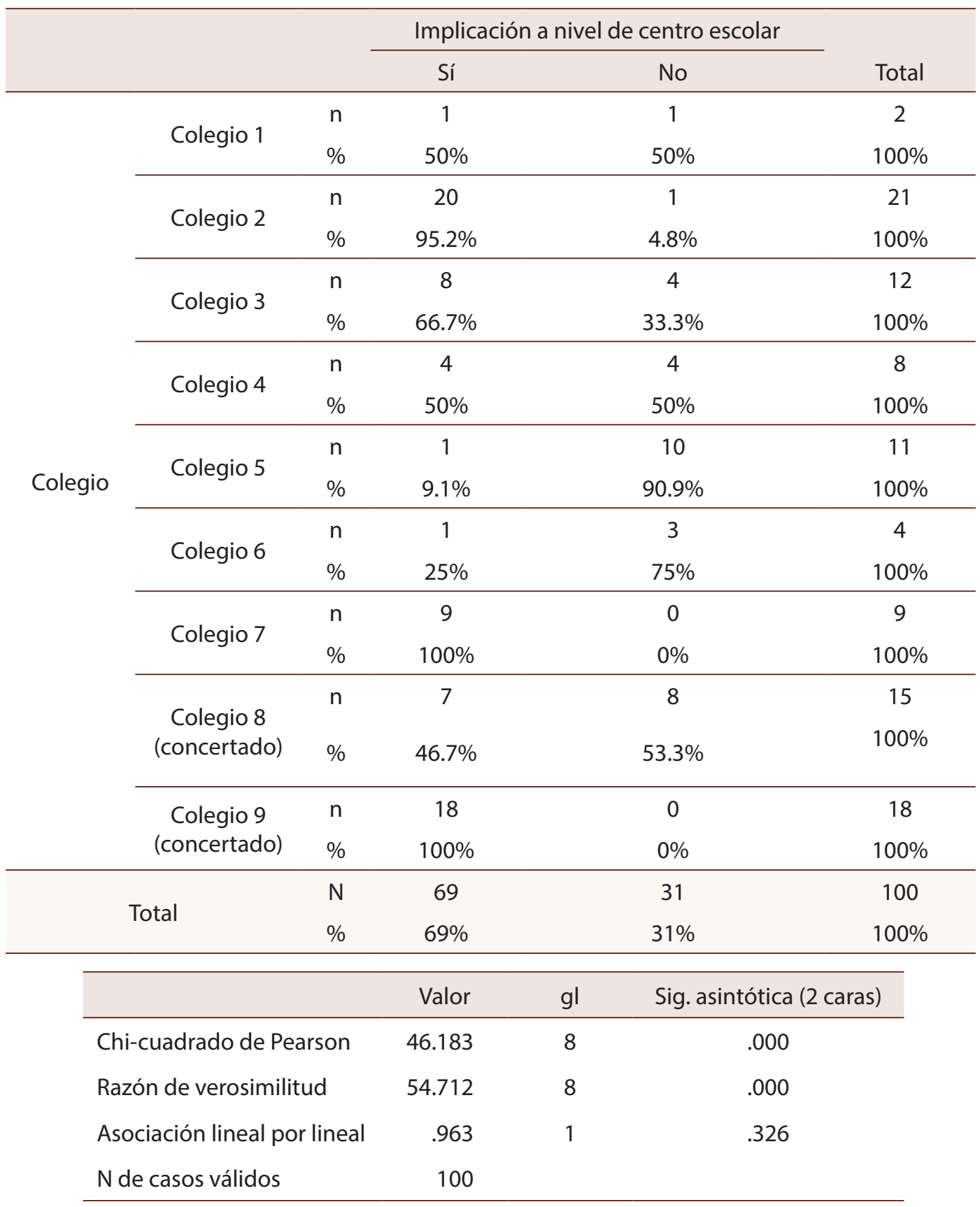

Nota: Elaboración propia. 
http://doi.org/10.15359/ree.25-3.6

\section{Discusión}

En la presente investigación se han analizado las respuestas de 100 profesionales de educación primaria en cuanto a sus conocimientos teóricos y experiencia profesional sobre la IE.

En primer lugar, respecto a la definición que la muestra hace de IE, se debe destacar que, en su mayoría, coincide plenamente con los hallazgos teóricos expuestos por referentes de la temática como Goleman (1995) o García-Fernández y Giménez-Mas (2010). Concretamente, 50 docentes, es decir, la mitad de la muestra, afirma que la IE es una capacidad o un conjunto de capacidades, aunque 19 también la definen como una habilidad o conjunto de habilidades.

De manera complementaria a los códigos basados en definir la IE como capacidad o habilidad, también se ha respondido a la pregunta utilizando algunas de las habilidades concretas que componen los modelos de IE comentados al inicio de este trabajo (Bar-On, 2006; García-Tudela y Marín-Sánchez, 2019; Rovira, 1998). Principalmente, la muestra se ha basado en aludir habilidades como la gestión emocional, la empatía o incluso de manera aislada, también hay casos que señalan la inteligencia intrapersonal o el autoconcepto.

Este hecho evidencia que un porcentaje significativo de la muestra, al menos, tiene una aproximación a lo que teóricamente es la IE, puesto que la ha definido como una capacidad o conjunto de capacidades o habilidades, entre las que han destacado algunas. A pesar de la tendencia positiva de definir adecuadamente la IE, también hay casos en los que se ofrece una visión reduccionista que cataloga el concepto como empatía, o también de manera aislada, códigos como motor del resto de inteligencias o el trabajo de las emociones, que se alejan totalmente de las definiciones referentes de IE.

Respecto a las respuestas que se han centrado en destacar la utilidad de la IE, sobre todo se ha señalado que un óptimo desarrollo de esta posibilita identificar y reconocer las emociones, regularlas, expresarlas, entre otras habilidades propias de la IE ya comentadas previamente. En menor medida, se señalan diferentes utilidades como tener una buena salud mental, aportar soluciones, adaptarse al medio, las cuales también estarían estrechamente relacionadas con otras aportaciones científicas (Martínez Sánchez, 2019; Ozáez Aguilar, 2015).

Si el foco de atención lo trasladamos a la práctica profesional, cabe destacar que no se han obtenido diferencias significativas entre trabajar cotidianamente la IE y las diferentes variables sociodemográficas que se han tenido en cuenta para este estudio, es decir, el sexo, la edad, la especialidad o el nivel educativo. Dicho de otro modo, el trabajo que se desarrolla en el aula en torno a la IE no depende directamente de ninguna condición de las expuestas; posiblemente, porque el nivel de IE docente en el contexto español suele ser adecuado y existe una conciencia colectiva en torno a la necesidad de trabajar la IE para mejorar la calidad de vida, ya no únicamente docente, sino también del alumnado (Aguayo-Muela y Aguilar-Luzón, 2017). 
http://doi.org/10.15359/ree.25-3.6

http://www.una.ac.cr/educare

educare@una.ac.cr

En cuanto a cómo se está trabajando formalmente la IE, es preciso destacar algunas respuestas: en asambleas o por medio del teatro, ya que son coincidentes con alguna de la bibliografía utilizada en este trabajo (Gómez Vicario et al., 2017; Morán Iranzo et al., 2016). Sin embargo, las personas encuestadas no hacen alusión expresa a la gamificación o al aprendizaje cooperativo, las cuales son opciones sobre las que existen diferentes referencias contextualizadas en primaria (Pozo-Rico y Sandoval, 2020; Rivera-Pérez et al., 2021). No obstante, si se tienen en cuenta los resultados vinculados al código libre materiales, se aprecia cómo el juego sí es un recurso utilizado por diferentes docentes; al igual que los cuentos, los cuales también son un óptimo recurso para trabajar las emociones desde las primeras edades (Cerezo Ros, 2021).

Muchas de las respuestas emitidas están relacionadas con lo que Aguaded Gómez y Valencia (2017) podrían definir como trabajar la IE de manera informal, es decir, sin que exista una clara intención y planificación de cómo poner en práctica las diferentes habilidades. Algunos de los códigos que evidencian este hecho son empatizar, motivación o consejos, etc. Consideramos que debe existir una dedicación explícita de la IE, es decir, que el alumnado y profesorado sean conscientes de que se están trabajando una serie de habilidades con el fin de mejorar su bienestar personal. El hecho de resolver aisladamente un problema en clase o de lograr una automotivación del alumnado solo por incluir recompensas durante una sesión no supone estar trabajando la IE. Especialmente porque, tal y como señala Sarrionandia y Garaigordobil (2017), el reto educativo actual consiste en implementar programas para favorecer el desarrollo socioemocional como el de Bañuls Bertomeu (2015), y no únicamente actividades puntuales y sin una intención clara y predefinida.

El hecho de alcanzar el horizonte mencionado es complicado, si no existe una involucración a nivel de todo el centro a partir de la que concretar planes, programas o proyectos para un desarrollo integral en todos los cursos educativos. Atendiendo a los resultados extraídos en este sentido, cabe destacar que un 31\% de la muestra reconoce que no existe una línea de actuación conjunta para toda la institución. La polarización evidente en estas respuestas resulta llamativa, puesto que en un mismo centro educativo conviven docentes que afirman tener unas iniciativas comunes para todo el colegio, mientras que hay otros grupos que exponen todo lo contrario. Tan solo hay dos centros educativos (colegio 7 y 9 ) en el que el $100 \%$ de sus docentes que participaron en la encuesta ha afirmado tener una iniciativa común. A partir del análisis cualitativo se puede afirmar que, al menos, estos centros coinciden internamente en que los proyectos que están trabajando son el proyecto en ti y el plan de salud en la escuela. Por el contrario, hay proyectos que solo se citan una vez, lo cual carece de coherencia interna, puesto que un proyecto común debería estar identificado por la totalidad de docentes de un mismo centro. Algunos ejemplos de estos casos son el proyecto el monstruo de colores, proyecto INNOVA, entre otros.

Por último, en torno a la formación profesional, cabe destacar que también es llamativo el hecho de que un 95\% afirme tener interés en formarse sobre la educación de la IE. Principalmente, porque es un signo que evidencia una necesidad docente que no está siendo cubierta con la 
http://doi.org/10.15359/ree.25-3.6

formación permanente o el reciclaje profesional que están recibiendo las personas encuestadas. Asimismo, este dato también podría ser un indicativo de lo necesaria que es la formación inicial del profesorado sobre la IE, es decir, la educación que se imparte a futuros y futuras docentes en las instituciones de educación superior, lo cual es una demanda sobre la que se ha estado escribiendo durante años (Bisquerra Alzina, 2005; García Domingo, 2021).

\section{Conclusiones}

A través de este estudio exploratorio sobre la IE dirigido a docentes de educación primaria de una ciudad de España, se ha determinado que una parte significativa de la muestra tiene una adecuada visión teórica del concepto de IE, así como de su utilidad. Asimismo, se evidencia que no existe correlación positiva entre las variables sociodemográficas y la práctica didáctica del tópico que tratamos.

En relación con las experiencias educativas, cabe destacar que se suelen desarrollar iniciativas concretas basadas en el teatro, las asambleas, etc.; pero también destacan numerosos casos en los que no existe una intención explícita de trabajar la IE, más bien la relacionan con un trabajo transversal a la práctica escolar, por ejemplo, por medio de resolver conflictos, empatizando, etc.

Teniendo en cuenta las conclusiones expuestas, se desarrollan las siguientes líneas futuras de investigación.

En primer lugar, un estudio descriptivo con una muestra más amplia y diversa a través del que se indague en otras variables sociodemográficas que no se han contemplado en esta investigación exploratoria, como la experiencia docente, la ratio de estudiantes por clase, etc.

En consonancia con esta idea también se propone un estudio correlacional donde a partir de los resultados extraídos de la práctica educativa, se investigue la relación que existe con el nivel de IE de su alumnado.

Además, totalmente vinculado a la colaboración dentro de los claustros y su cohesión a la hora de implementar el trabajo en el aula, sería adecuada la realización de un grupo focal con docentes o entrevistas a equipos directivos para indagar en las iniciativas comunes de educación emocional.

Por último, destacamos el diseño de un estudio experimental que permita analizar la efectividad de una intervención formativa extendida en el tiempo para docentes, y compararla con los efectos obtenidos en el aula.

\section{Declaración de Material complementario}

Este artículo tiene disponible, como material complementario:

-La versión preprint del artículo en https://doi.org/10.5281/zenodo.4042028 
http://doi.org/10.15359/ree.25-3.6

http://www.una.ac.cr/educare

educare@una.ac.cr

\section{Referencias}

Aguaded Gómez, M. C. y Valencia, J. (2017). Estrategias para potenciar la inteligencia emocional en educación infantil. Aplicación del modelo de Mayer y Salovey. Tendencias pedagógicas, 30, 175-190. https://doi.org/10.15366/tp2017.30.010

Aguayo-Muela, Á. del C. y Aguilar-Luzón, M. C. (2017). Principales resultados de investigación sobre inteligencia emocional en docentes españoles. Reidocrea, 6, 170-193. 10.30827/ Digibug.45497

Alam, A. y Ahmad, M. (2018). The role of teachers' emotiocnal intelligence in enhancing student achievement. Journal of Asia Business Studies, 12(1), 31-43. https://doi.org/10.1108/jabs08-2015-0134

Bañuls Bertomeu, R. (2015). Promoviendo la inteligencia emocional en la escuela. Diseño y evaluación del programa EDI [Tesis doctoral]. Universitat do València. https://bit. ly/2S2LOGV

Bar-On, R. (2006). The Bar-On model of emotional-social Intelligence (ESI). Psicothema, 18(Supp. 1), 13-25. http://bit.ly/2ZZQu4F

Bisquerra Alzina, R. (2005). La educación emocional en la formación del profesorado. Revista Interuniversitaria de Formación del Profesorado, 19(3), 95-114. http://bit.ly/2sLwXZD

Bisquerra Alzina, R. (2011). Educación emocional. Revista Padres y Maestros, 337, 5-8. http://bit. ly/302gY5x

Caicedo Cavagnis, E. y Zalagar-Jaime, M. F. (2018). Entrevistas cognitivas: Revisión, directrices de uso y aplicación en investigaciones psicológicas. Avaliação Psicológica, 17(3), 362-370. https://doi.org/10.15689/ap.2018.1703.14883.09

Casino-García, A. M., Llopis-Bueno, M. J. y Llinares-Insa, L. I. (2021). Emotional intelligence profiles and self-esteem/self-concept: An analysis of relationships in gifted students. International Journal of Environmental Research and Public Health, 18(3), 1-23. https://doi.org/10.3390/ ijerph18031006

Cerezo Ros, M. del C. (2021). Efectos del trabajo con cuentos motores en educación infantil sobre el control inhibitorio y emociones. Lecturas: Educación Física y Deportes, 25(273), 128-140. https://doi.org/10.46642/efd.v25i273.2329

Chen, J. y Guo, W. (2020). Emotional intelligence can make a difference: The impact of principals' emotional intelligence on teaching strategy mediated by instructional leadership. Educational Management Administration \& leadership, 48(1), 82-105. https://doi. org/10.1177/1741143218781066 
http://doi.org/10.15359/ree.25-3.6

http://www.una.ac.cr/educare educare@una.ac.cr

Collins, D. (2003). Pretesting survey instruments: An overview of cognitive methods. Quality of Life Research, 12(3), 229-238. https://doi.org/10.1023/A:1023254226592

Damasio, A. R. (1994). Descartes's error. Emotion reason, and the human brain. AVON Books. http:// bit.ly/2T4tf8a

Dell'Angela, L., Zaharia, A., Lobel, A., Vico Begara, O., Sander, D. y Samson, A. C. (2020). Board games on emotional competences for school-age children. Games for health journal, 9(3), 187-196. https://doi.org/10.1089/g4h.2019.0050

Fernández-Díez, J., Cencerrado-de-Aller, R., Rodríguez-Fernández, J., Hierro-Berceo, Á., SánchezFernández, E.y Pérez-Sáenz, J. (2017).“Explora”: Atender a los alumnos con más curiosidad, motivación o capacidad sin excluir a nadie. Psicología Educativa, 23(2), 95-103. https://doi. org/10.1016/j.pse.2017.05.004

Galdón López, S. y Melguizo Ibañez, E. (2019). Educating emotionally the students of the third cycle of elementary grade. Education, Sport, Health and Physical Activity (ESHPA): International Journal, 3(3), 428-435. https://bit.ly/3eGsM6h

García Domingo, B. (2021). Competencia emocional en maestros de educación infantily primaria. Fuentes de variabilidad y sugerencias de mejora. Revista Electrónica Interuniversitaria de Formación del Profesorado, 24(2), 1-15. https://doi.org/10.6018/reifop.450111

García-Fernández, M. y Giménez-Mas, S. I. (2010). La inteligencia emocional y sus principales modelos: Propuesta de un modelo integrador. Espiral. Cuadernos del Profesorado, 3(6), 4352. https://doi.org/10.25115/ecp.v3i6.909

García-Retana, J. Á. (2012). La educación emocional, su importancia en el proceso de aprendizaje. Revista Educación, 36(1), 97-109. https://doi.org/10.15517/revedu.v36i1.455

García-Tudela, P.A. y Marín-Sánchez, P. (2019). Educación en inteligencia emocional y social: Revisión y propuesta teórico-didáctica. Revista de Estudios e Investigación en Psicología y Educación, 6(1), 68-83. https://doi.org/10.17979/reipe.2019.6.1.5167

Goleman, D. (1995). Emotional intelligence. Bantam Books.

Goleman, D. (1998). Working with emotional intelligence. Bantam.

Gómez Vicario, M., Velasco Martínez, L. C. y Tójar Hurtado, J. C. (2017). Proyecto de intervención sobre educación emocional en la comunidad El Milagro (Iquitos, Perú). Cuestiones pedagógicas: Revista de Ciencias de la Educación, 26, 111-130. https://bit.ly/3bB3NPZ

Hassan, A., Pinkwart, N. y Shafi, M. (2021). Serious games to improve social and emotional intelligence in children with autism. Entertainment computing, 38(1), 100417. https://doi. org/10.1016/j.entcom.2021.100417 
http://doi.org/10.15359/ree.25-3.6

http://www.una.ac.cr/educare

educare@una.ac.cr

Hernández Sampieri, R., Fernández Collado, C. y Baptista Lucio, M. del P. (2010). Metodología de

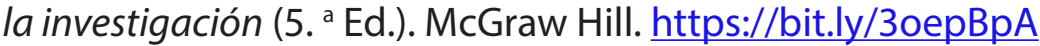

Martínez Sánchez, A. (2019). Competencias emocionales y rendimiento académico en los estudiantes de educación primaria. Psychology, Society, \& Education, 11(1), 15-25. https:// doi.org/10.25115/psye.v111i1.1874

Massot, I., Dorio, I. y Sabariego, M. (2016). Estrategias de recogida y análisis de la información (5. ${ }^{a}$ ed.). En R. Bisquerra (Ed.), Metodología de la investigación educativa (pp. 321-357). La Muralla. https://bit.ly/2XNDhfl

Mayer, J. D. y Salovey, P. (1997). What is emotional intelligence? En P. Salovey y D. J. Sluyter (Eds.), Emotional development and emotional intelligence: Educational implications (pp. 3-34). Basic Books.

Meneses, J. (2016). El cuestionario. Universitat Oberta de Catalunya. https://bit.ly/3uV75Fh

Mora, F. (2013). ¿Qué es una emoción? Arbor, 189(759), 1-6. https://doi.org/10.3989/ arbor.2013.759n1003

Morán Iranzo, J. T., Ruiz Díaz, M. E. y Marín Suelves, D. (2016). El teatro, un recurso para el desarrollo de competencias y para la inclusión. Aula de innovación educativa, 248, 39-43. https://bit. ly/3yl6q28

Ozáez Aguilar, M. T. (2015). Inteligencia emocional en educación primaria. Revista Internacional de Apoyo a la Inclusión, Logopedia, Sociedad y Multiculturalidad, 1(3), 51-60. http://bit. ly/37J1W7k

Pastor-Gil, L. y Blázquez-Saiz, I. (2019). Evaluación de los programas de educación emocional ante situaciones de acoso escolar. Revista Electrónica en Educación y Pedagogía, 3(5), 22-38. https://doi.org/10.15658/rev.electron.educ.pedagog19.09030502

Pozo-Rico, T. y Sandoval, I. (2020). Can academic achievement in primary school students be improved through teacher training on emotional intelligence as a key academic competency? Frontiers in Psychology, 10, 1-8. https://doi.org/10.3389/fpsyg.2019.02976

Rivera-Pérez, S., Fernández-Rio, J. e Iglesias-Gallego, D. (2021). Effects of an 8-week cooperative learning intervention on physical education student's task and self-approach goals, and emotional intelligence. International Journal of Environmental Research and Public Health, 18(1), 1-11. https://doi.org/10.3390/ijerph18010061

Rosas, O. (2011). La estructura disposicional de los sentimientos. Ideas y valores, 60(145), 5-31. http://bit.ly/2uu07gh 
Rovira, F. (1998). Com saber si un és emocionalment intel.ligent? Aloma, 2, 57-68. http://bit. ly/36BbCk5 https://dialnet.unirioja.es/servlet/articulo?codigo=261728

Sabariego Puig, M. (2004). El proceso de investigación. En R. Bisquerra Akzina (Coord.), Metodología de la investigación educativa (5. a ed., pp. 267-284). La Muralla. https://bit. ly/2XNDhfl

Salas, N., Alcaide, M. y Hurtado, A. (2018). Programas de intervención en inteligencia emocional para educación infantil. Revistas de estilos de aprendizaje, 11(22), 137-165. https://bit. ly/2AY6F9Z

Salovey, P. y Mayer, J. D. (1990). Emotional intelligence. Imagination, Cognition and Personality, 9(3), 185-211. https://doi.org/10.2190/DUGG-P24E-52WK-6CDG

Sánchez Ortega, A. G. y Chacón-Cuberos, R. (2021). Estudio de la inteligencia emocional en escolares de educación primaria. Alcance en el área de educación física. Sport TK: Revista Euroamericana de Ciencias del Deporte, 10(1), 87-93. https://doi.org/10.6018/sportk.461701

Sarrionandia, A. y Garaigordobil, M. (2017). Efectos de un programa de inteligencia emocional en factores socioemocionales y síntomas psicosomáticos. Revista Latinoamericana de Psicología, 49(2), 110-118. https://doi.org/10.1016/j.rlp.2015.12.001

Torrado Fonseca, M. (2009). Estudios de encuesta (5. a ed.). En R. Bisquerra Alzina (Coord.), Metodología de la investigación educativa (pp. 231-257). La Muralla. https://bit.ly/2XNDhfl

Torrego-Seijo, J. C., Caballero-García, P. Á., Lorenzo-Llamas, E. M. (2020). The effects of cooperative learning on trait emotional intelligence and academic achievement of spanish primary school students. British Journal of Educational Psychology. https://doi.org/10.1111/bjep.12400

Uitto, M., Jokikokko, K. y Estola, E. (2015). Virtual special issue on teachers and emotions in Teaching and Teacher Education (TATE) in 1985-2014. Teaching and Teacher Education, 50, 124-135. https://doi.org/10.1016/j.tate.2015.05.008

Valente, S.y Lourenço, A. A. (2020). Conflict in the classroom:How teachers'emotional intelligence influences conflict management. Frontiers in education, 5, 1-10. https://doi.org/10.3389/ feduc.2020.00005

Vázquez, C. y López-Larrosa, S. (2016). Resultados de la aplicación de un programa para la mejora de la inteligencia emocional en alumnos de 4. ${ }^{\circ}$ de primaria. En J. L. Soler, L. Aparicio, O. Díaz, E. Escolano y A. Rodríguez (Coords.), Inteligencia emocional y bienestar II. Reflexiones, experiencias profesionales e investigaciones (pp. 807-816). Ediciones Universidad de San Jorge. http://bit.ly/37MWK2q 